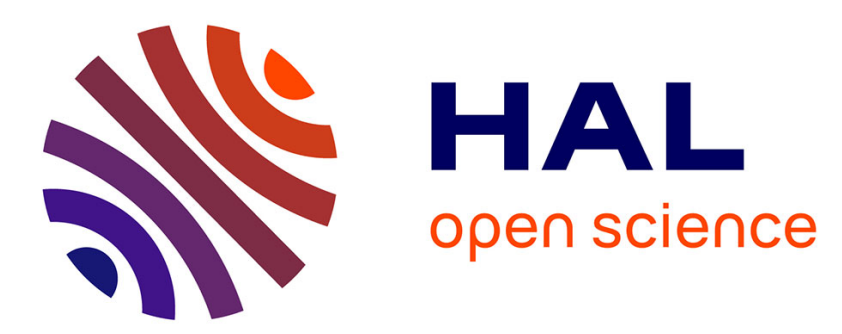

\title{
A look at the turbulent wake using scale-by-scale energy budgets
}

\author{
F. Thiesset, L. Danaila, R.A. Antonia
}

\section{To cite this version:}

F. Thiesset, L. Danaila, R.A. Antonia. A look at the turbulent wake using scale-by-scale energy budgets. Lecture Notes in Mechanical Engineering, 2014, 8, pp.3-16. 10.1007/978-3-642-40371-2_1. hal-01612113

\section{HAL Id: hal-01612113 \\ https://hal.science/hal-01612113}

Submitted on 28 Mar 2019

HAL is a multi-disciplinary open access archive for the deposit and dissemination of scientific research documents, whether they are published or not. The documents may come from teaching and research institutions in France or abroad, or from public or private research centers.
L'archive ouverte pluridisciplinaire HAL, est destinée au dépôt et à la diffusion de documents scientifiques de niveau recherche, publiés ou non, émanant des établissements d'enseignement et de recherche français ou étrangers, des laboratoires publics ou privés. 


\title{
A look at the turbulent wake using scale-by-scale energy budgets
}

\author{
F. Thiesset, L. Danaila and R.A. Antonia
}

\begin{abstract}
It is now well established that coherent structures exist in the majority of turbulent flows and can affect various aspects of the dynamics of these flows, such as the way energy is transferred over a range of scales as well as the departure from isotropy at the small scales. Reynolds and Hussain (1972) were first to derive onepoint energy budgets for the coherent and random motions respectively. However, at least two points must be considered to define a scale and allow a description of the mechanisms involved in the energy budget at that scale. A transport equation for the second-order velocity structure function, equivalent to the Karman-Howarth (1938) equation for the two-point velocity correlation function, was written by Danaila, Anselmet, Zhou \& Antonia (1999) and tested in grid turbulence, which represents a reasonable approximation to (structureless) homogeneous isotropic turbulence. The equation has since been extended to more complicated flows, for example the centreline of a fully developed channel flow and the axis of a self-preserving circular jet. More recently, we have turned our attention to the intermediate wake of a circular cylinder in order to assess the effect of the coherent motion on the scale-byscale energy distribution. In particular, energy budget equations, based on phaseconditioned structure functions, have revealed additional forcing terms, the most important of which highlights an additional cascade mechanism associated with the coherent motion. In the intermediate wake, the magnitude of the maximum energy transfer clearly depends on the nature of the coherent motion.
\end{abstract}

Key words: Coherent structure, Scale-by-scale budget, Wake Flow

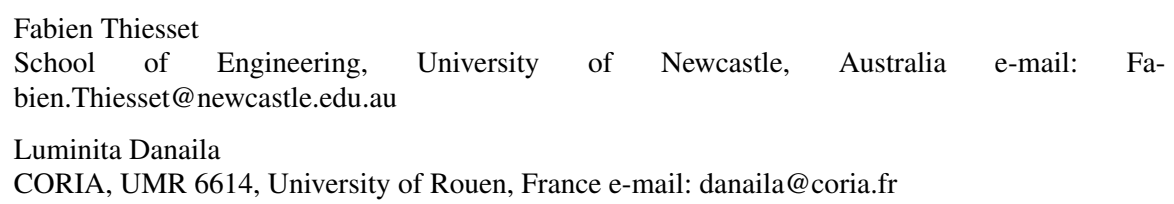




\section{Introduction}

Turbulent flows give rise to a wide and continuous range of scales. The largest eddies reflect the way the kinetic energy is injected in the system and therefore depend on the type of flow. In contrast, one frequently asserts that the anisotropic and nonuniversal influence of the largest scales diminishes during the first non-linear local interactions and is thus expected to decline at the smallest scales. Consequently, it is often postulated that the smallest scales have the best prospect of being universal or quasi-universal ([19]), this paradigm being usually related to its initiator [14].

Recently, [12] argued that neither the original derivation of the Kolmogorov 2/3 and 4/5 laws, nor all the subsequent derivations of the 4/5 law use the assumption of locality of interactions and the existence of a cascade. Another analysis is provided by [10] who demonstrated that the 4/5 law implies the statistical dependence of the difference and sum of velocities at two points separated by a distance $r$, the sum being a measure of the large scale motion $([10,15])$. This analytical treatment was then accurately confirmed by the experiments of [12] in different high Reynolds number flows. Hence, we now dispose of very strong theoretical and experimental evidences that the way energy is distributed between different scales results from direct and bidirectional non-local interactions. The key argument in favour of the statistical universality of the smallest scales is thus worth investigating even at very high Reynolds number flows.

Moreover, the Reynolds number needed for the Kolmogorov theory to hold is usually beyond the reach of laboratory experiments. Consequently, large and small scales interact within a common range of scales, and remain correlated one with each other.

Because of the pioneering work of [23] and of many other researchers, it is now well known that most shear flows give rise to so-called coherent structures. These are energy containing eddies at rather large scales, but in contrast with classical largescale turbulence, coherent structures strongly persist in time and/or in space. Their topology depends on initial conditions and their related statistics are not universal $([2,22])$. The information enclosed within coherent structures persists till the far field $([3,26,22])$ and may thus influence small-scale statistics due to non-local interactions and finite Reynolds numbers effects.

Therefore, some new statistical tools must be developed to quantify the interactions between large (and in particular the CM) and small scales. To this end, one possible way is to consider velocity increments, which represent motions at scales $\leq r$ (not only at the particular scale $r[15,8]$ ), conditioned by another parameter characterizing motions at scales $\geq r([15])$.

The first study based on conditional structure functions is that of [17]. The latter reported structure functions conditioned by the instantaneous velocity in two different high Reynolds number shear flows. They pointed out a strong correlation between large and inertial subrange scales. These authors [17] finally concluded that these findings are in contradiction with the random sweeping decorrelation which was first stated ([21]) by supposing statistical independence between eddies. 
Subsequently, measured structure functions were conditioned by the instantaneous velocity in the atmospheric boundary layer [20]. In spite of the very high Reynolds number, a strong correlation between large and small scales also emerged. According to [20], this result may highlight the persisting influence of the mean shear on the smallest scales.

More recently, [5, 4] measured structure functions conditioned by the average of the velocity at points $x$ and $x+r$ in different shearless flows. These authors pointed out that small scales were perturbed by the large scales at very different levels depending on the flow type. They concluded that conditional structure functions provide a reference tool for comparing large-scale effects in different flows.

Investigating the nature of the interactions between the coherent motion (CM) and the small scale motion is the principal motivation of the present work. A better understanding of the effect of the large-scale coherent motion on the smallest scales is of major practical interest, for example for designing new efficient sub-grid scale models $([16,11])$. The present study addresses two specific issues: (i) Does the kinetic energy at a scale $r$ depend on the dynamics of the CM? (ii) What are the energy budget equations based on phase-conditioned structure functions?

To unravel these issues, the way we condition structure functions is somewhat different to that presented previously. Based on the approach of e.g. [18], we propose to condition structure functions by a particular value of the phase $\phi$ arising from the phase-averaging operation.

This study focuses entirely on a circular cylinder wake flow, which is investigated by means of hot wire experiments. The wake flow is renowned for its coherent motion which persists far downstream from the obstacle (see e.g. [3]). Different streamwise locations were studied, from $x=10 D$ to $x=40 D$ ( $D$ is the cylinder diameter) leading to a decreasing amplitude of velocity coherent fluctuations. Investigations are mostly oriented on the wake centerline, for which the mean shear is absent. This allows us to focus only on the influence of the coherent motion, thus avoiding the additive effect of the mean shear ([20]).

The paper is organized as follows. First, measurements are described. Then, onepoint statistics are presented, with particular emphasis on the typical topology of the wake flow. Afterwards, the interactions between the coherent and the random fluctuating fields are highlighted by means of second-order phase-conditioned structure functions. Finally, energy budget equations which concern both the random and coherent motions are derived and tested against experimental data.

\section{Experiments}

The reader can refer to [25] for more detailed information. We recall briefly the most important features of the experimental set-up and measurement technique.

Measurements were carried out in an open-circuit wind tunnel with a working section of $0.35 \times 0.35 \mathrm{~m}^{2}$ and $2.4 \mathrm{~m}$ long. The cylinder of diameter $D=12.7 \mathrm{~mm}$ is placed horizontally, spanning the full width of the working section. The upstream 
velocity $U_{0}$ is $3 m s^{-1}$ corresponding to a Reynolds number $R_{D}=\frac{U_{0} D}{v}=2540(v$ is the kinematic viscosity). Measurements were made at different downstream locations: $x=10,20,40 D$, and different transverse positions, from $y=0$ to $y=3 D$. The Taylor micro-scale Reynolds number $R_{\lambda}=\frac{\sqrt{u^{2}} \lambda}{v} \approx 70$ at $40 D \cdot \overline{u^{2}}$ is the streamwise velocity variance, $\lambda$ is the Taylor micro-scale $\lambda^{2}=15 v \overline{u^{2}} / \bar{\varepsilon}$ and $\bar{\varepsilon}$ the mean dissipation rate (see [25] for the values and the procedure employed for measuring $\bar{\varepsilon}$ ). A hot-wire probe, consisting of four cross-wires was used to measure simultaneously all three vorticity components (see [25] for more details about the probe).

Phase-averaged statistics are obtained as follows. The transverse velocity component $v$ is first digitally band-pass filtered at the Strouhal frequency, using an eighthorder Butterworth filter. The filtering operation is next applied to the magnitude of the Fourier transform of $v$ in order to avoid any phase shift. Then, the Hilbert transform $h$ of the filtered signal $v_{f}$ is obtained and the phase $\phi$ inferred from the relation $\phi=\arctan \left(\frac{h}{v_{f}}\right)$. Finally, the phase is divided into 41 segments and phaseaveraged statistics are calculated for each segment. The convergence of statistics was checked, by reducing the number of segments, and found to be satisfactory. By means of our method, phase-averaged quantities are calculated over the period $[-\pi, \pi]$. As was done by [16], the phase is doubled up to $[-2 \pi, 2 \pi]$ thanks to the periodicity, in order to enhance the visual display.

In [9], the geometrical space (location $\mathbf{x}$ in the flow) and the separation space (turbulent scales $\mathbf{r}$ ) are made independent by considering the geometrical location specified by the midpoint $\mathbf{X}=\frac{1}{2}\left(\mathbf{x}+\mathbf{x}^{+}\right)$with $\mathbf{x}^{+}=\mathbf{x}+\mathbf{r}$. The same idea is applied here to phase-conditioned structure functions for which the phase $\phi$ is defined as the phase at the midpoint $\phi=\phi(\mathbf{X})$. Therefore, each velocity component is decomposed into a triple contribution from the mean temporal average, the phase-averaged fluctuation and the random/turbulent fluctuation.

\section{Flow Topology}

Let us first briefly discuss the overall features of the coherent motion pertaining to the wake flow.

One of the main advantages of using phase averaging is that the temporal dynamics associated with the presence of the CM is highlighted. As far as the wake flow is concerned, one generally displays statistics in the $(\phi, y)$ plane $([26,3])$ to relate the spatial organization of the kinetic energy to that of the coherent structures. Here, we focus particularly on the coherent strain which is generally enrolled in the creation of turbulent kinetic energy.

Iso-values of the coherent strain $\tilde{S}=\left(\frac{\partial \tilde{u}}{\partial y}+\frac{\partial \tilde{v}}{\partial x}\right)$ are reported in Figs. 1(a), 1(b), 1 (c). Note that $\phi=\pi / 2 \pm k \pi$ and $y=0$ corresponds to the location of the maximum (absolute values) of the coherent strain. Its amplitude decreases further downstream 


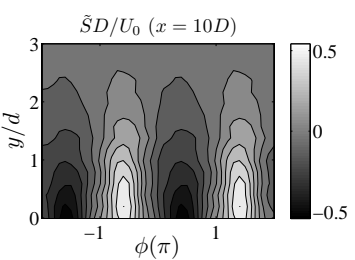

(a)

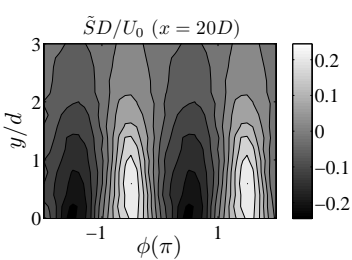

(b)

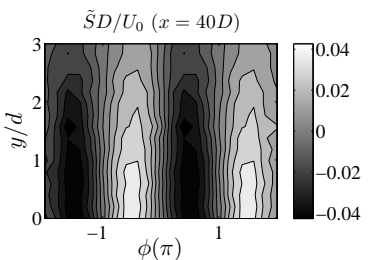

(c)

Fig. 1 Coherent strain $\tilde{S}=\left(\frac{\partial \tilde{u}}{\partial y}+\frac{\partial \tilde{v}}{\partial x}\right)$ normalized by $D / U_{0}$ in the $(\phi, y)$ plane. (a) $x=10 D$, (b) $x=20 D$, (c) $x=40 D$

from the obstacle. For example, the extrema of $\tilde{S}$ at $40 D$ are one order of magnitude smaller than at $10 D$.

In the light of the previous remarks, in the remaining part of this study, we will focus entirely on the wake centerline, where the contribution of the coherent motion is maximum. In addition, this allows the effect of the coherent motion to be separated from that of the mean shear. The latter is known to influence the behaviour of the smallest scales even at large Reynolds numbers ([17, 20, 12]).

\section{Phase-conditioned structure functions}

The phase-averaging operation is now applied to the $n-t h$ order structure functions. Structure functions are as usual functions of $r$, but specific to our methodology, they are also functions of the phase $\phi$. In the following, we assign brackets to phaseconditioned structure functions, $v i z$.

$$
\left\langle\left(\Delta u_{\alpha}\right)^{n}\right\rangle(r, \phi)=\left\langle\left(u_{\alpha}(x+r)-u_{\alpha}(x)\right)^{n}\right\rangle(r, \phi)
$$

which represent the ensemble average of the $n-t h$ order increment $\Delta u_{\alpha}=u_{\alpha}(x+$ $r)-u_{\alpha}(x)$ of the velocity component $u_{\alpha}$, for a particular value of $\phi$. Then, the conventional time-averaged structure functions are calculated by integrating $\left\langle(\Delta u)^{n}\right\rangle$ over all values of $\phi \in[-\pi ; \pi]$ (periodicity is invoked), and are denoted by a horizontal bar, i.e. $\overline{\left\langle\left(\Delta u_{\alpha}\right)^{n}\right\rangle}$. An important remark is that for $n=1$,

$$
\left\langle\Delta u_{\alpha}\right\rangle=\Delta \tilde{u}_{\alpha} \neq 0 .
$$

Therefore, in contrast to classical structure functions, first-order phase-conditioned structure functions are non-zero for $r \neq 0$. However, $\overline{\left\langle\Delta u_{\alpha}\right\rangle}=0$. Another result relates to the second-order structure functions,

$$
\left\langle\left(\Delta u_{\alpha}\right)^{2}\right\rangle=\left(\Delta \tilde{u}_{\alpha}\right)^{2}+\left\langle\left(\Delta u_{\alpha}^{\prime}\right)^{2}\right\rangle
$$




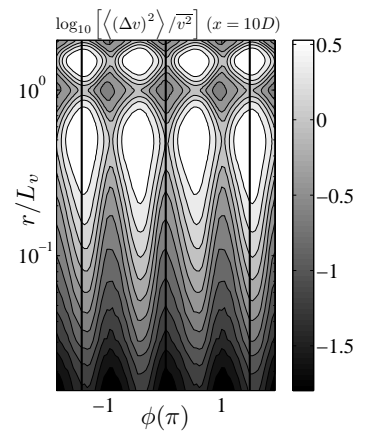

(a)

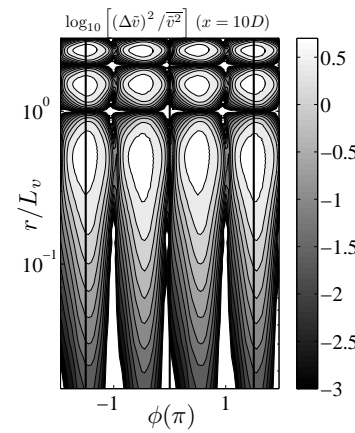

(d)

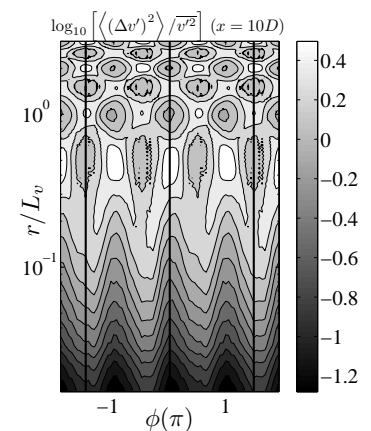

(g)

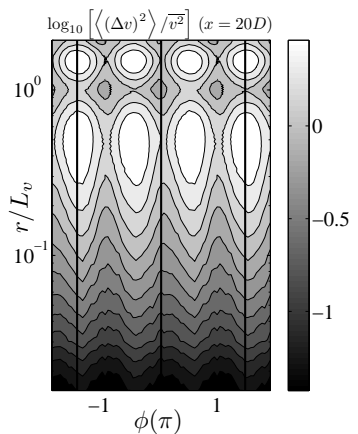

(b)

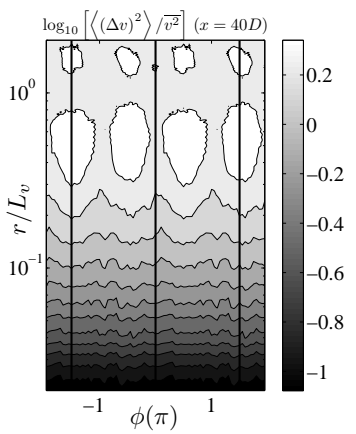

(c)

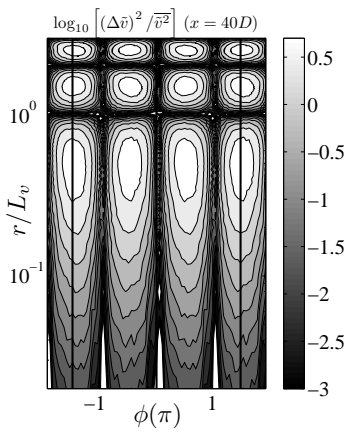

(f)

(e)

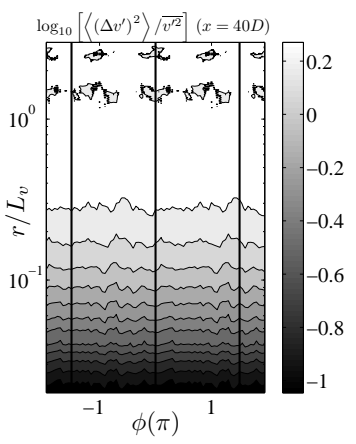

(i)

Fig. 2 Phase-conditioned second-order structure function of the transverse velocity component $v$ (using $\log _{10}$ scale). (a , b, c) $:\left\langle(\Delta v)^{2}\right\rangle / \overline{v^{2}}$. (d, e, f) $:(\Delta \tilde{v})^{2} / \overline{\tilde{v}^{2}}$. (g, h, i ) $:\left\langle\left(\Delta v^{\prime}\right)^{2}\right\rangle / \overline{v^{\prime 2}}$. (a, d, g) $x=10 D,(\mathrm{~b}, \mathrm{e}, \mathrm{h}) x=20 D$, (c, f, i) $x=40 D$. Vertical solid lines delineate phase references $\phi= \pm \frac{3 \pi}{2}$ and $\phi=0$.

i.e. the sum of the second-order structure functions of the random and coherent motions. The influence of the phase, on the total, organized or random fluctuating fields, can be thus assessed separately. 


\subsection{Focus on the transverse velocity component}

We present the phase-conditioned structure functions of the total transverse velocity fluctuations (Figs. 2(a), 2(b), 2(c)), of the coherent component (Figs. 2(d), 2(e), 2(f)), and of the randomly fluctuating field (Figs. 2(g), 2(h), 2(i)) for three downstream distances.

In homogeneous turbulence, the second-order structure function should be equal to twice the velocity variance at large scales. In Figs. 2(a), 2(b), 2(c), the values of the phase-conditioned second-order structure functions progressively increase as $r$ keeps increasing, and reach a maximum for $r=L_{v} / 2\left(L_{v}\right.$ is the distance between two successive vortices) which overshoots $2 \overline{v^{2}}$. Then, one notes a slight decrease of energy up to $r=L_{v}$ and this pattern is repeated with a period equal to $L_{v}$.

The influence of the phase (hereafter called phase-correlation) is clearly visible in Figs. 2(a), 2(b), 2(c). The energy of the transverse velocity component $v$ is largest at phases $\phi=\frac{\pi}{2} \pm k \pi$ with a periodicity of $\pi$. These phase locations and periodicity are strongly linked to the maxima of the coherent shear (Fig.1). Note finally that at $\mathrm{x}=40 D$ the phase-correlation strongly diminishes at smallest scales, although it is still perceptible for scales larger than $r=0.2 L_{v} \approx 2 \lambda$.

The phase-conditioned structure function of $v$ exhibits a topology analogous to that of $\tilde{v}$ (Figs. 2(d), 2(e), 2(f)). For example, the phase $\pi$-periodicity and the separation $L_{v}$-periodicity are recovered. Further, the local maxima or minima corresponds to that of the coherent motion. Therefore, as for one-point statistics, the energy distribution among scales of the total fluctuating field hides a non negligible contribution due to the presence of the coherent motion, which tends to decrease as we move away from the obstacle.

Using Eq.(3), we can calculate the structure function of the random velocity component $v^{\prime}$ (Figs. 2(g), 2(h), 2(i)). At $\mathrm{x}=40 \mathrm{D}$, the phase-conditioned structure functions have similar shapes to the usual time-average structure functions, and the phase-correlation is lost. However, at $\mathrm{x}=10 \mathrm{D}$ and $20 \mathrm{D}$, there is still a strong influence of the phase, even at very small scales.

\section{Energy budget equations}

In flows where a phase-correlation at a given scale is strongly discernible, it might be interesting to derive an energy budget equation for second-order phase-conditioned structure function. One advantage of using the phase-average operation is that it allows writing transport equations for the two distinct energy contributions: that of the coherent motion and that of the purely random/turbulent part of the fluctuating field. The next section aims at deriving such kinds of budget equations. 


\subsection{General formulation}

The starting point is the Navier-Stokes equation

$$
\frac{\partial U_{i}}{\partial t}+U_{j} \frac{\partial U_{i}}{\partial x_{j}}=-\frac{\partial P}{\partial x_{i}}+v \frac{\partial^{2} U_{i}}{\partial x_{j} \partial x_{j}},
$$

where $P$ is the the kinematic pressure and $v$ the kinematic viscosity. In contrast to [6], [7], we invoke the triple decomposition ([18])

$$
\begin{array}{rr}
U_{i}=\bar{U}_{i}+\tilde{u}_{i}+u_{i}^{\prime}, & P=\bar{P}+\tilde{p}+p^{\prime} \\
u_{i}=\tilde{u}_{i}+u_{i}^{\prime}, & p=\tilde{p}+p, \\
\left\langle u_{i}^{\prime}\right\rangle=0, & \left\langle p^{\prime}\right\rangle=0 .
\end{array}
$$

By further using Eq. 5, [18] obtained the dynamical equations of the coherent and random components, which are respectively

$$
\begin{gathered}
\frac{D \tilde{u}_{i}}{D t}+\tilde{u}_{j} \frac{\partial \bar{U}_{i}}{\partial x_{j}}+\frac{\partial}{\partial x_{j}}\left(\tilde{u}_{i} \tilde{u}_{j}-\overline{\tilde{u}_{i} \tilde{u}_{j}}\right)+\frac{\partial}{\partial x_{j}}\left(\left\langle u_{i}^{\prime} u_{j}^{\prime}\right\rangle-\overline{u_{i}^{\prime} u_{j}^{\prime}}\right)=-\frac{\partial \tilde{p}}{\partial x_{i}}+v \frac{\partial^{2} \tilde{u}_{i}}{\partial x_{j} \partial x_{j}},(6) \\
\frac{D u_{i}^{\prime}}{D t}+\tilde{u}_{j} \frac{\partial u_{i}^{\prime}}{\partial x_{j}}+u_{j}^{\prime} \frac{\partial \bar{U}_{i}}{\partial x_{j}}+u_{j}^{\prime} \frac{\partial \tilde{u}_{i}}{\partial x_{j}}+\frac{\partial}{\partial x_{j}}\left(u_{i}^{\prime} u_{j}^{\prime}-\left\langle u_{i}^{\prime} u_{j}^{\prime}\right\rangle\right)=-\frac{\partial p^{\prime}}{\partial x_{i}}+v \frac{\partial^{2} u_{i}^{\prime}}{\partial x_{j} \partial x_{j}} .(7)
\end{gathered}
$$

$\frac{D}{D t}=\frac{\partial}{\partial t}+\overline{U_{j}} \frac{\partial}{\partial x_{j}}$ is the material derivative. Eqs. 6 and 7 are written at points $\mathbf{x}$ and $\mathbf{x}^{+}=\mathbf{x}+\mathbf{r}$ separated by a distance $\mathbf{r}$. Then, the equation at point $\mathbf{x}$ is subtracted to that at point $\mathbf{x}^{+}$, so that

$$
\begin{gathered}
\frac{\partial \Delta \tilde{u}_{i}}{\partial t}+\Delta\left(\bar{U}_{j} \frac{\partial \tilde{u}_{i}}{\partial x_{j}}\right)+\Delta\left(\tilde{u}_{j} \frac{\partial \bar{U}_{i}}{\partial x_{j}}\right)+\Delta\left(\frac{\partial}{\partial x_{j}}\left(\tilde{u}_{i} \tilde{u}_{j}-\overline{\tilde{u}_{i} \tilde{u}_{j}}\right)\right) \\
+\Delta\left(\frac{\partial}{\partial x_{j}}\left(\left\langle u_{i}^{\prime} u_{j}^{\prime}\right\rangle-\overline{u_{i}^{\prime} u_{j}^{\prime}}\right)\right)=-\Delta\left(\frac{\partial \tilde{p}}{\partial x_{i}}\right)+v \Delta\left(\frac{\partial^{2} \tilde{u}_{i}}{\partial x_{j}^{2}}\right)
\end{gathered}
$$

is the dynamical equation of coherent velocity increments, and

$$
\begin{aligned}
\frac{\partial \Delta u_{i}^{\prime}}{\partial t}+ & \Delta\left(\bar{U}_{j} \frac{\partial u_{i}^{\prime}}{\partial x_{j}}\right)+\Delta\left(\tilde{u}_{j} \frac{\partial u_{i}^{\prime}}{\partial x_{j}}\right)+\Delta\left(u_{j}^{\prime} \frac{\partial \bar{U}_{i}}{\partial x_{j}}\right)+\Delta\left(u_{j}^{\prime} \frac{\partial \tilde{u}_{i}}{\partial x_{j}}\right) \\
& +\Delta\left(\frac{\partial}{\partial x_{j}}\left(u_{i}^{\prime} u_{j}^{\prime}-\left\langle u_{i}^{\prime} u_{j}^{\prime}\right\rangle\right)\right)=-\Delta\left(\frac{\partial p^{\prime}}{\partial x_{i}}\right)+v \Delta\left(\frac{\partial^{2} u_{i}^{\prime}}{\partial x_{j}^{2}}\right)
\end{aligned}
$$

is the dynamical equation of random velocity increments.

As proposed by [9], [6], [7], we use derivatives with respect to the midpoint $\mathbf{X}$ defined by $\mathbf{X}=\left(\mathbf{x}+\mathbf{x}^{+}\right) / 2$, such as $\partial / \partial x_{j}=-\partial / \partial r_{j}+\partial / 2 \partial X_{j}$ and $\partial / \partial x_{j}^{+}=$ $\partial / \partial r_{j}+\partial / 2 \partial X_{j}$. We further assume the two points $\mathbf{x}$ and $\mathbf{x}^{+}$are independent ([9], [6], [7]), viz. $\partial u_{i} / x_{j}^{+}=\partial u_{i}^{+} / x_{j}=0$. After multiplying Eq. 8 by $2 \Delta \tilde{u}_{i}$ and Eq. 9 by 
$2 \Delta u_{i}^{\prime}$, applying phase averaging followed by time averaging, and finally noting that

$$
\begin{aligned}
& \left\langle\Delta u_{j}^{\prime} \Delta u_{i}^{\prime}\right\rangle=\left\langle\Delta u_{i} \Delta u_{j}\right\rangle-\Delta \tilde{u}_{i} \Delta \tilde{u}_{j} \\
& \left\langle\Delta u_{j} \Delta q^{2}\right\rangle=\Delta \tilde{u}_{j} \Delta \tilde{q}^{2}+\Delta \tilde{u}_{j}\left\langle\Delta q^{\prime 2}\right\rangle+\left\langle\Delta u_{j}^{\prime} \Delta q^{\prime 2}\right\rangle+2 \Delta \tilde{u}_{i}\left\langle\Delta u_{j}^{\prime} \Delta u_{i}^{\prime}\right\rangle,
\end{aligned}
$$

we finally obtain the scale-by-scale energy budget of the coherent motion

$$
\begin{aligned}
& \overline{\frac{D}{D t} \Delta \tilde{q}^{2}}+\frac{1}{2} \frac{\partial}{\partial X_{j}}\left[\overline{\Sigma \tilde{u}_{j} \Delta \tilde{q}^{2}}+2 \overline{\left\langle\Sigma u_{j}^{\prime} \Delta u_{i}^{\prime}\right\rangle \Delta \tilde{u}_{i}}+2 \overline{\Delta \tilde{u}_{i} \Delta \tilde{p}}\right]+2 \overline{\Delta \tilde{u}_{i} \Delta \tilde{u}_{j}} \frac{\partial \bar{U}_{i}}{\partial x_{j}} \\
& -\overline{\left\langle\Sigma u_{j}^{\prime} \Delta u_{i}^{\prime}\right\rangle \frac{\partial}{\partial X_{j}} \Delta \tilde{u}_{i}}+\frac{\partial}{\partial r_{j}} \overline{\Delta \tilde{u}_{j} \Delta \tilde{q}^{2}}+2 \overline{2 \Delta \tilde{u}_{i} \frac{\partial}{\partial r_{j}}\left\langle\Delta u_{i}^{\prime} \Delta u_{j}^{\prime}\right\rangle} \\
& -v\left[\left(2 \frac{\partial^{2}}{\partial r_{j}^{2}}+\frac{1}{2} \frac{\partial^{2}}{\partial X_{j}^{2}}\right) \overline{\Delta \tilde{q}^{2}}+2 \Sigma\left(\frac{\partial \tilde{u}_{i}}{\partial x_{j}} \frac{\partial \tilde{u}_{j}}{\partial x_{i}}\right)\right]=-2 \Sigma \overline{\tilde{\varepsilon}}
\end{aligned}
$$

For the random motion, the corresponding energy budget equation is

$$
\begin{array}{r}
\overline{\frac{D}{D t} \Delta q^{\prime 2}}+\frac{1}{2} \frac{\partial}{\partial X_{j}}\left[\overline{\Sigma u_{j}^{\prime} \Delta q^{\prime 2}}+\overline{\Sigma \tilde{u}_{j}\left\langle\Delta q^{\prime 2}\right\rangle}+2 \overline{\Delta u_{i}^{\prime} \Delta p^{\prime}}\right]+2 \overline{\Delta u_{i}^{\prime} \Delta u_{j}^{\prime}} \frac{\partial \bar{U}_{i}}{\partial x_{j}} \\
+\overline{\left\langle\Sigma u_{j}^{\prime} \Delta u_{i}^{\prime}\right\rangle \frac{\partial}{\partial X_{j}} \Delta \tilde{u}_{i}}+\frac{\partial}{\partial r_{j}}\left(\overline{\left\langle\Delta u_{j} \Delta q^{2}\right\rangle}-\overline{\Delta \tilde{u}_{j} \Delta \tilde{q}^{2}}\right)-2 \Delta \tilde{u}_{i} \frac{\partial}{\partial r_{j}}\left\langle\Delta u_{i}^{\prime} \Delta u_{j}^{\prime}\right\rangle \\
-v\left[\left(2 \frac{\partial^{2}}{\partial r_{j}^{2}}+\frac{1}{2} \frac{\partial^{2}}{\partial X_{j}^{2}}\right) \overline{\Delta q^{\prime 2}}+2 \Sigma\left(\frac{\partial u_{i}^{\prime}}{\partial x_{j}} \frac{\partial u_{j}^{\prime}}{\partial x_{i}}\right)\right]=-2 \Sigma \overline{\varepsilon^{\prime}}
\end{array}
$$

$\overline{\Delta \tilde{q}^{2}}=\overline{\Delta \tilde{u}_{i} \Delta \tilde{u}_{i}}$ and $\overline{\Delta q^{\prime 2}}=\overline{\Delta u_{i}^{\prime} \Delta u_{i}^{\prime}}$ (the summation convention applies to repeated Latin indices) are the coherent and random kinetic energies at a given scale respectively. $\Sigma \bullet=\bullet(\mathbf{x}+\mathbf{r})+\bullet(\mathbf{x})$ is the sum at two points separated by a distance $\mathbf{r}$. The quantities $\tilde{\varepsilon}=\frac{v}{2} \overline{\left(\frac{\partial \tilde{u}_{i}}{\partial x_{j}}+\frac{\partial \tilde{u}_{j}}{\partial x_{i}}\right)^{2}}$ and $\varepsilon^{\prime}=\frac{v}{2} \overline{\left(\frac{\partial u_{i}^{\prime}}{\partial x_{j}}+\frac{\partial u_{j}^{\prime}}{\partial x_{i}}\right)^{2}}$ are the mean energy dissipation rates of the coherent and the random motions, respectively.

For the sake of clarity, these equations are formally rewritten as

$$
\begin{array}{r}
\mathscr{I}_{c}+\mathscr{A}_{c m}+\mathscr{D}_{c c}+\mathscr{D}_{r c}^{1}+\mathscr{D}_{c p}+\mathscr{P}_{c m}-\mathscr{P}_{r c}+\mathscr{T}_{c}+\mathscr{F}_{c}+\mathscr{V}_{c}=-2 \Sigma \overline{\tilde{\varepsilon}} \\
\mathscr{I}_{r}+\mathscr{A}_{r m}+\mathscr{D}_{r r}+\mathscr{D}_{r c}^{2}+\mathscr{D}_{r p}+\mathscr{P}_{r m}+\mathscr{P}_{r c}+\mathscr{T}-\mathscr{T}_{c}-\mathscr{F}_{c}+\mathscr{V}_{r}=-2 \Sigma \overline{\bar{\varepsilon}^{\prime}}
\end{array}
$$

where $\mathscr{I}, \mathscr{A}, \mathscr{D}, \mathscr{P}, \mathscr{T}, \mathscr{F}$ et $\mathscr{V}$ denote respectively the non stationarity, advection, diffusion, production, transfer, forcing and viscous terms. The subscripts $m, c, r$ correspond to the mean, coherent and random motions, and $\mathscr{D}_{p}$ indicates the pressure diffusion.

By comparison with [6],[7], there are additional terms which emerge in the present equations, e.g. the terms $\mathscr{P}_{r c}, \mathscr{T}_{c}$ and $\mathscr{F}_{c}$ which can be identified as the production of random fluctuations by the coherent motion, the coherent kinetic energy transfer and the forcing associated by the presence of a coherent motion. All 
three are present in equations 12 and 13, but with opposite signs. This means that what represents a loss of energy for the coherent motion Eq. 12, constitutes a gain of energy for randomly fluctuating motion (Eq. 13). Further, we can shed light on $\mathscr{D}_{r c}^{1}$ and $\mathscr{D}_{r c}^{2}$, the transport of random statistical quantities by the organized motion.

Equations (12) and (13) provide a general framework which allow the physics of the interaction between coherent and random fields to be unravelled.

In a globally homogeneous context, the limit at the largest scales of Eqs. (12) and (13) is twice the one-point energy budgets provided by [18]. At this stage, Eqs. 12 and 13 are functions of the time $t$, the reference point vector $\mathbf{x}$ and the separation vector $\mathbf{r}$. This leads to a problem in 7 dimensions ( 8 before time-averaging). In order to reduce the number of degrees of freedom, one generally invokes the local isotropy assumption. The practical consequence is that these isotropic forms lead themselves being tested experimentally, with the constraint that two-points statistics are usually evaluated along one particular direction. Further, this allows us to compare the present considerations to that already developed over the last half century ([13], [24], [1], [6], [7]).

\subsection{Locally homogeneous and isotropic context}

First, if homogeneity holds at the level of the viscous scales, then the viscous term reduces to ([9])

$$
-v\left[\left(2 \frac{\partial^{2}}{\partial r_{j}^{2}}+\frac{1}{2} \frac{\partial^{2}}{\partial X_{j}^{2}}\right) \overline{\Delta q^{\prime 2}}+2 \Sigma\left(\overline{\frac{\partial u_{i}^{\prime}}{\partial x_{j}} \frac{\partial u_{j}^{\prime}}{\partial x_{i}}}\right)\right]=-2 v \frac{\partial^{2}}{\partial r_{j}^{2}} \overline{\Delta q^{\prime 2}},
$$

Then, in the context of local isotropy, the divergence and the Laplacian operators are expressed in spherical coordinates ([6], [7]), and after multiplying (12) and (13) by $r^{2}=r_{j} r_{j}$, integrating with respect to $r$ and dividing by $r^{2}$, we obtain

$$
\begin{array}{r}
\frac{1}{r^{2}} \int_{0}^{r} s^{2}\left(\mathscr{A}_{c m}+\mathscr{D}_{c c}+\mathscr{D}_{r c}^{1}+\mathscr{P}_{c m}+\mathscr{P}_{r c}+\mathscr{D}_{c p}\right) d s \\
+\overline{\Delta \tilde{u}_{\|} \Delta \tilde{q}^{2}}+\frac{2}{r^{2}} \int_{0}^{r} \overline{\Delta \tilde{u}_{i} \frac{\partial}{\partial s} s^{2}\left\langle\Delta u_{\|}^{\prime} \Delta u_{i}^{\prime}\right\rangle} d s-2 v \frac{\partial}{\partial r} \overline{\Delta \tilde{q}^{2}}=-\frac{4}{3} \overline{\tilde{\varepsilon}} r \\
+\overline{\left\langle\Delta u_{\|} \Delta q^{2}\right\rangle}-\overline{\Delta \tilde{u}_{\|} \Delta \tilde{q}^{2}}-\frac{2}{r^{2}} \int_{0}^{r} \overline{\Delta \tilde{u}_{i} \frac{\partial}{\partial s} s^{2}\left\langle\Delta u_{\|}^{\prime} \Delta u_{i}^{\prime}\right\rangle} d s-2 v \frac{\partial}{\partial r} \overline{\Delta q^{\prime 2}}=-\frac{4}{3} \overline{\varepsilon^{\prime}} r .
\end{array}
$$

Eqs. (17) and (18) are the energy budget equations for the coherent and random components in a locally isotropic context. Here, $s$ is a dummy variable and the subscript 
$\|$ denotes the direction parallel to the separation vector. When the spatial separation is inferred using Taylor hypothesis, this direction coincides with that of the mean flow.

The first line of Eqs. (17) and (18) represents the energy contribution of the largest scales ([6], [7]). The main difference with respect to the extended form of Kolmogorov's equation [1], is the appearance of several extra terms due to the presence of CM. The effective energy transfer of the random velocity component is explicit and thus consists of the total energy transfer $\overline{\left\langle\Delta u_{\|} \Delta q^{2}\right\rangle}$ (including the coherent and random contributions), from which are subtracted the coherent energy transfer $\overline{\Delta \tilde{u}_{\|} \Delta \tilde{q}^{2}}$ and the forcing term $\frac{2}{r^{2}} \int_{0}^{r} \overline{\Delta \tilde{u}_{i} \frac{\partial}{\partial s} s^{2}\left\langle\Delta u_{\|}^{\prime} \Delta u_{i}^{\prime}\right\rangle} d s$.

\subsection{Comparison with experiments}

On the wake centerline, the locally isotropic scale-by-scale budget of the random motion is

$$
\begin{array}{r}
-\frac{1}{r^{2}} \int_{0}^{r} s^{2} \mathscr{A}_{r m} d s-\overline{\left\langle\Delta u_{\|} \Delta q^{2}\right\rangle} \\
+\overline{\Delta \tilde{u}_{\|} \Delta \tilde{q}^{2}}+\frac{2}{r^{2}} \int_{0}^{r} \overline{\Delta \tilde{u}_{i} \frac{\partial}{\partial s} s^{2}\left\langle\Delta u_{\|}^{\prime} \Delta u_{i}^{\prime}\right\rangle} d s+2 v \frac{\partial}{\partial r} \overline{\Delta q^{\prime 2}}=\frac{4}{3} \overline{\varepsilon^{\prime}} r
\end{array}
$$

which means that in the limit of large scales, the advection term is almost entirely compensated by the energy dissipation rate, other large scale terms such as longitudinal production or turbulent diffusion are negligible compared to the advection term.

Fig. 3(a) reports the total non linear transfer $-\overline{\left\langle\Delta u_{\|} \Delta q^{2}\right\rangle}$, the additional coherent transfer and forcing due to the coherent motion $\overline{\Delta \tilde{u}_{\|} \Delta \tilde{q}^{2}}+\frac{2}{r^{2}} \int_{0}^{r} \overline{\Delta \tilde{u}_{i} \frac{\partial}{\partial s} s^{2}\left\langle\Delta u_{\|}^{\prime} \Delta u_{i}^{\prime}\right\rangle} d s$ and the effective transfer inferred from their sum, in function of the separation $r / L_{v}$.

For weakly turbulent flows the non linear transfer term is smaller than $\frac{4}{3} \varepsilon r$, because of the cross-over between viscous and large-scale effects ([6], [7]). Here, $-\overline{\left\langle\Delta u_{\|} \Delta q^{2}\right\rangle} / \varepsilon r \approx 0.63$. The additional energy transfer associated with the coherent motion is negative, its value being quite small, although non negligible. Its contribution is non zero for all separations, with a maximum contribution at about $2 \lambda$. Finally, the maximum effective transfer of the random motion is smaller than the total transfer by about $12 \%$.

Even though the difference of $12 \%$ between the total energy transfer and the effective energy transfer is quite weak, its influence may persist far from the injection of energy ([22]) and thus remains a key point to provide some insight into the energy transfer along the cascade in the wake flow, for which the CM is discernible.

The extra-transfer term is non zero over a large range of scales. However, one cannot claim that this quantifies the non local interactions between coherent and 


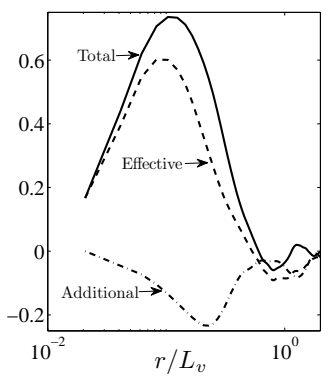

(a)

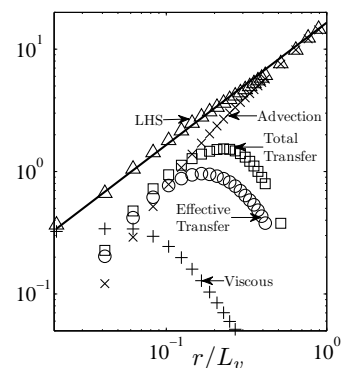

(b)

Fig. 3 (a) Non linear transfer term divided by $\overline{\varepsilon^{\prime}} r$. —— total transfer term $-\overline{\left\langle\Delta u_{\|} \Delta q^{2}\right\rangle},-$ - coherent transfer and forcing term $\overline{\Delta \tilde{u}_{\|} \Delta \tilde{q}^{2}}+\frac{2}{r^{2}} \int_{0}^{r} \overline{\Delta \tilde{u}_{i} \frac{\partial}{\partial s} s^{2}\left\langle\Delta u_{\|}^{\prime} \Delta u_{i}^{\prime}\right\rangle} d s,-\cdots-\operatorname{Sum}-\overline{\left\langle\Delta u_{\|} \Delta q^{2}\right\rangle}+$ $\overline{\Delta \tilde{u}_{\|} \Delta \tilde{q}^{2}}+\frac{2}{r^{2}} \int_{0}^{r} \overline{\Delta \tilde{u}_{i} \frac{\partial}{\partial s} s^{2}\left\langle\Delta u_{\|}^{\prime} \Delta u_{i}^{\prime}\right\rangle} d s$. (c) Scale-by-scale budget of the random motion Eq. (19) divided by $\overline{\varepsilon^{\prime}}$. — : $: \frac{4}{3} \overline{\varepsilon^{\prime}} r, \times:-\frac{1}{r^{2}} \int_{0}^{r} s^{2} \mathscr{A}_{r m} d s, \square-\overline{\left\langle\Delta u_{\|} \Delta q^{2}\right\rangle}, \bigcirc:-\overline{\left\langle\Delta u_{\|} \Delta q^{2}\right\rangle}+\overline{\Delta \tilde{u}_{\|} \Delta \tilde{q}^{2}}+$ $\frac{2}{r^{2}} \int_{0}^{r} \overline{\Delta \tilde{u}_{i} \frac{\partial}{\partial s} s^{2}\left\langle\Delta u_{\|}^{\prime} \Delta u_{i}^{\prime}\right\rangle} d s,+: 2 v \frac{\partial}{\partial r} \overline{\Delta q^{\prime 2}}, \Delta$ Left hand side of Eq. (19).

random fields because of the loss of localization in physical space as mentioned previously.

The balance between the right-and left-hand sides of (19) is reasonably satisfied at all scales. The weak imbalance at rather large scales appears to reflect that local isotropy no more holds or that another production and/or diffusion terms must taken into account in Eq. 19.

To conclude, the experimental investigation in the cylinder intermediate wake supports the analytical considerations provided by this study.

\section{Conclusions}

The connection between the temporal dynamics of the coherent motion and the energy distribution across all scales is highlighted by means of phase-conditioned structure functions. This original statistical tool allows us to assess what range of scales is affected by the CM dynamics. Moreover, we are able to separate the energy contributions of the coherent and random fluctuations. Phase-averaging the structure functions measured in a cylinder wake yields two main outcomes.

First, it is shown that, as the downstream location behind the cylinder increases, i) the scale at which the forcing associated with the $\mathrm{CM}$ is perceptible continually increases; ii) phase-scale structure functions reveal also that a scale $r$ is correlated with that of the coherent shear, the effect of the latter being to locally enhance the energy at any scale $r$. 
Second, energy budget equations which account for the organized motion are derived. Both general and isotropic formulations are obtained. They highlight a few additional terms. One of these may be interpreted as an additional forcing exerted by the $\mathrm{CM}$ on the random motion. At $\mathrm{x}=40 \mathrm{D}$, this term represents only about $12 \%$ of the total transfer term, but its influence may persist far downstream. The isotropic formulation is tested against experimental data at the wake centreline. The weak imbalance between the analytical formulation and the measurements at rather large scales appears to underline the inadequacy of local isotropy at these scales.

Acknowledgements The financial support of the 'Agence Nationale de la Recherche' (ANR), under the project 'ANISO', is gratefully acknowledged. RAA acknowledges the support of the Australian Research Council. We thank T. Zhou who carried out the original experiments.

\section{References}

1. R. A. Antonia, M. Ould-Rouis, F. Anselmet, and Y. Zhu. Analogy between predictions of Kolmogorov and Yaglom. J. Fluid Mech., 332:395-409, 1997.

2. R. A. Antonia, T. Zhou, and G. P. Romano. Small-scale turbulence characteristics of two dimensional bluff body wakes. J. Fluid Mech., 459:67-92, 2002.

3. D. K. Bisset, R. A. Antonia, and L. W. B. Browne. Spatial organization of large structures in the turbulent far wake of a cylinder. J. Fluid Mech., 218:439-461, 1990.

4. D. B. Blum, G. P. Bewley, E. Bodenschatz, M. Gibert, A. Gylfason, L. Mydlarski, G. A. Voth, $\mathrm{H}$. Xu, and P. K. Yeung. Signatures of non-universal large scales in conditional structure functions from various turbulent flows. New J. Phys., 13:113020, 2011.

5. D. B. Blum, S. B. Kunwar, J. Johnson, and G. A. Voth. Effects of nonuniversal large scales on conditional structure functions in turbulence. Phys. Fluids, 22:015107, 2010.

6. L. Danaila, F. Anselmet, and R. A. Antonia. An overview of the effect of large scale inhomogeneities on small-scale turbulence. Phys. Fluids, 14:2475-2484, 2002.

7. L. Danaila, R. A. Antonia, and P. Burattini. Progress in studying small-scale turbulence using 'exact' two-point equations. New J. Phys., 6:128, 2004.

8. L. Danaila, R. A. Antonia, and P. Burattini. Comparison between kinetic energy and passive scalar energy transfer in locally homogeneous isotropic turbulence. Physica D, 241:224-231, 2012.

9. R. J. Hill. Equations relating structure functions of all orders. J. Fluid Mech., 434:379-388, 2001.

10. I. Hosokawa. A paradox concerning the refined similarity hypothesis of Kolmogorov for isotropic turbulence. Prog. Theor. Phys., 118:169, 2007.

11. H. S. Kang and C. Meneveau. Universality of large eddy simulation model parameters across a turbulent wake behind a heated cylinder. J. Turb., 3:1-27, 2002.

12. M. Kholmyansky and A. Tsinober. Kolmogorov 4/5 law, nonlocality, and sweeping decorrelation hypothesis. Phys. Fluids, 20:041704, 2008.

13. A. Kolmogorov. Dissipation of energy in the locally isotropic turbulence. Dokl. Akad. Nauk. SSSR, 125:15-17, 1941.

14. A. Kolmogorov. The local structure of turbulence in incompressible viscous fluid for very large Reynolds numbers. Proc. USSR Ac. of Sci. (Trad. Proc Roy. Soc London 434 1991)., 30:299-303, 1941.

15. H. Mouri and A. Hori. Two-point velocity average of turbulence: Statistics and their implications. Phys. Fluids, 22:115110, 2010.

16. J. O'Neil and C. Meneveau. Subgrid-scale stresses and their modelling in a turbulent plane wake. J. Fluid Mech., 349:253-293, 1997. 
17. A. A. Praskovsky, E. Gledzer, M. Y. Karyakin, and Y. Zhou. The sweeping decorrelation hypothesis and energy-inertial range interaction in high Reynolds number flow. J. Fluid Mech., 248:493-511, 1993.

18. W. C. Reynolds and A. K. M. F. Hussain. The mechanics of an organised wave in turbulent shear flow. J. Fluid Mech., 54:263-288, 1972.

19. K. R. Sreenivasan and R. A. Antonia. The phenomenology of small-scale turbulence. Ann. Rev. Fluid Mech., 29:435-472, 1997.

20. K. R. Sreenivasan and B. Dhruva. Is there a scaling in high Reynolds-number turbulence? Prog. Theor. Phys., 130:103, 1998.

21. H. Tennekes. Eulerian and Lagrangian time microscales in isotropic turbulence. J. Fluid Mech., 67:561-567, 1975.

22. F. Thiesset, L. Danaila, and R. A. Antonia. Dynamical effect of the total strain induced by the coherent motion on local isotropy in a wake. J. Fluid Mech., 720:393-423, 2013.

23. A. A. Townsend. The structure of turbulent shear flows. Cambridge University Press, 1956.

24. A. M Yaglom. On the local structure of a temperature field in a turbulent flow. Dokl. Akad. Nauk SSSR, 69:743, 1949.

25. T. Zhou, Y. Zhou, M.W Yiu, and L.P Chua. Three-dimensional vorticity in a turbulent cylinder wake. Exp. Fluids, 35:459-471, 2003.

26. Y. Zhou and R. A. Antonia. Memory effects in a turbulent plane wake. Exp. Fluids, 19:112$120,1995$. 